\title{
Hemorrhage in mouse tumors induced by dodecaborate cluster lipids intended for boron neutron capture therapy
}

This article was published in the following Dove Press journal:

International Journal of Nanomedicine

29 July 2014

Number of times this article has been viewed

\author{
Tanja Schaffran' \\ Nan Jiang' \\ Markus Bergmann ${ }^{2,3}$ \\ Ekkehard Küstermann ${ }^{4}$ \\ Regine Süss ${ }^{5}$ \\ Rolf Schubert ${ }^{5}$ \\ Franz MWagner 6 \\ Doaa Awad ${ }^{7}$ \\ Detlef Gabel ${ }^{1,2,8}$ \\ 'Department of Chemistry, \\ University of Bremen, ${ }^{2}$ Institute \\ of Neuropathology, Klinikum \\ Bremen-Mitte; ${ }^{3}$ Cooperative \\ Center Medicine, University of \\ Bremen, "“In-vivo-MR" AG, FB2, \\ University of Bremen, Bremen, \\ ${ }^{5}$ Pharmaceutical Technology, \\ University of Freiburg, Freiburg im \\ Breisgau, ${ }^{6}$ Forschungsneutronenquelle \\ Heinz Maier-Leibnitz (FRM II), \\ Technische Unversitaet Muenchen, \\ Garching, Germany; ${ }^{7}$ Department of \\ Biochemistry, Alexandria University, \\ Alexandria, Egypt; ${ }^{8} \mathrm{School}$ of \\ Engineering and Science, Jacobs \\ University Bremen, Bremen, Germany
}

Correspondence: Detlef Gabel Jacobs University Bremen, Campus Ring I, D-28579 Bremen, Germany Tel +49 42I 2003585

Fax +49 42I 2003249

Email d.gabel@jacobs-university.de
Abstract: The potential of boron-containing lipids with three different structures, which were intended for use in boron neutron capture therapy, was investigated. All three types of boron lipids contained the anionic dodecaborate cluster as the headgroup. Their effects on two different tumor models in mice following intravenous injection were tested; for this, liposomes with boron lipid, distearoyl phosphatidylcholine, and cholesterol as helper lipids, and containing a polyethylene glycol lipid for steric protection, were administered intravenously into tumor-bearing mice $(\mathrm{C} 3 \mathrm{H}$ mice for SCCVII squamous cell carcinoma and BALB/c mice for CT26/WT colon carcinoma). With the exception of one lipid (B-THF-14), the lipids were well tolerated, and no other animal was lost due to systemic toxicity. The lipid which led to death was not found to be much more toxic in cell culture than the other boron lipids. All of the lipids that were well tolerated showed hemorrhage in both tumor models within a few hours after administration. The hemorrhage could be seen by in vivo magnetic resonance and histology, and was found to occur within a few hours. The degree of hemorrhage depended on the amount of boron administered and on the tumor model. The observed unwanted effect of the lipids precludes their use in boron neutron capture therapy.

Keywords: liposomes, histology, magnetic resonance imaging, pharmacodynamics

\section{Introduction}

Boron neutron capture therapy (BNCT) is potentially a very effective method for the treatment of localized tumors. ${ }^{1,2}$ For this therapy to be successful, boron-10 must be accumulated in concentrations of more than 10-20 ppm in the target tissue. ${ }^{3,4}$ For the therapy to be successful, the chemical form of the boron compounds, and their possible binding to components of the blood and tissues, are of little importance as long as there is a sufficient amount of boron in the target tissue. As the amount of boron in the tumor should be as high as possible, compounds should contain boron units with as many boron atoms as possible. This is best achieved with tumor targeting compounds containing boron clusters, such as the o-carborane $\left(\mathrm{B}_{10} \mathrm{C}_{2}\right)$, nido-carborane $\left(\mathrm{B}_{9} \mathrm{C}_{2}[1-]\right)$ or closo-dodecaborate $\left(\mathrm{B}_{12}[2-]\right)$ clusters. The latter two are ionic, whereas the former is electrically neutral and very hydrophobic.

Up to now, only two substances are in clinical trials for BNCT, namely p-dihydroxyborylphenylalanine and $\mathrm{Na}_{2} \mathrm{~B}_{12} \mathrm{H}_{11} \mathrm{SH}(\mathrm{BSH})$. However, neither of these have optimal accumulation properties in tumors, dihydroxyborylphenylalanine accumulation being limited and short-lived and BSH accumulation being not very selective, except in brain tumors. Hence, they are not optimal boron delivery agents. Better compounds are therefore very much sought after. In order to possess potential in BNCT, their toxicity should be low so that enough boron can be delivered for successful therapy. 
Recently, a number of research groups, including the current authors, have concentrated on the synthesis of lipids containing ionic boron clusters as polar head groups. ${ }^{5-11}$ The lipids should be ideal carriers of boron when incorporated into liposomes, especially when the liposomes carry a targeting unit such as transferrin or other suitable agents. ${ }^{12}$ Liposomes made from boron-containing lipids can carry at least the same amount of boron as lipids into which watersoluble boron compounds are encapsulated. ${ }^{8}$

Li et $\mathrm{al}^{11}$ have investigated one lipid with a nidocarborane headgroup and found that its in vivo toxicity was sufficiently severe to make this lipid of limited if any use for BNCT. Nakamura et al found, for the same headgroup, that liposomes were not stable when the boron lipid was present in $50 \%$ mol. ${ }^{13}$ Furthermore, the lipid was found to be toxic in vivo. ${ }^{14}$ For both lipids, no data on cytotoxicity are available.

The authors have recently synthesized three types of boron lipids, which all carry the dodecaborate cluster as headgroup but have different linkers and frameworks to which hydrophobic chains are attached, and investigated their cytotoxicity against mammalian cells..$^{8-10}$ Here, the effect of these boron cluster lipids was studied in two different mouse models, with the aim of checking in vivo toxicity and tumor accumulation by the enhanced permeation and retention effect. ${ }^{15}$ One lipid was found to be toxic when given in amounts relevant for BNCT. The other two types of lipids led to rapid hemorrhage selectively in the tumor. Magnetic resonance imaging (MRI) measurements were performed to visualize the development of hemorrhage in the tumor tissue after injection of liposomes.

\section{Materials and methods Liposome preparation}

Distearoylphosphatidylcholine (DSPC) and 1,2-distearoyl-snglycero-3-phosphoethanolamine-N-(methoxy[polyethylene glycol]-2000) (DSPE-PEG) were from Lipoid GmbH (Ludwigshafen, Germany). Cholesterol was purchased from Acros Organics (Geel, Belgium).

The boron lipids that were used have been described previously: B-6-14, ${ }^{8}$ THF-S- $12,{ }^{10}$ THF-S- $16,{ }^{10}$ and B-THF-14. ${ }^{9}$ Details about the preparation and analytics of the lipids and their incorporation into liposomes can be found in the respective citations. Their cytotoxicity in V79 cells is shown in Table 1. Liposomes were prepared from mixtures of DSPC, cholesterol, and the boron lipid as indicated in the Results section, together with $2 \% \mathrm{~mol}$ (calculated based on the total amount of lipid) DSPE-PEG
Table I Cytotoxicity of the boron lipids tested

\begin{tabular}{ll}
\hline Boron lipid & $\begin{array}{l}\text { Cytotoxicity (median effective } \\
\text { dose }[\mathrm{mM}] \text { in V79 cells) }\end{array}$ \\
\hline B-6- $14^{8}$ & 3.9 \\
B-THF- $14^{9}$ & 0.38 \\
THF-S- $12^{10}$ & 1.5 \\
THF-S- $16^{10}$ & 4.8 \\
Dioxan-S- $16^{10}$ & 3.0 \\
\hline
\end{tabular}

Notes: Data from Justus et al ${ }^{8}$ and Schaffran et al. ${ }^{9}$ Adapted with permission from Schaffran T, Burghardt A, Barnert S, et al. Pyridinium lipids with the dodecaborate cluster as polar headgroup: synthesis, characterization of the physical-chemical behavior, and toxicity in cell culture. Bioconjug Chem. 2009;20(II):2190-2198. Copyright $\odot 2009$ American Chemical Society. ${ }^{10}$

by hydration, five freeze-thaw cycles, and extrusion (21 times through a $100 \mathrm{~nm}$ filter) in HEPES-buffered saline solution $(\mathrm{pH}=7.4)$.

The $\zeta$-potential and the size was measured as described by Justus et al. ${ }^{8}$

\section{Tumor models}

Mice (BALB/c and $\mathrm{C} 3 \mathrm{H})$ were purchased from Charles River GmbH (Sulzfeld, Germany). Tumor cells were implanted subcutaneously into the ventral skin or, for MRI, into the right hind leg. For BALB/c, CT26.WT cells (CRL-2638; American Type Culture Collection, Manassas, VA) were used. For C3H, SCCVII cells (provided by Dr Ono; Kyoto University Research Reactor Institute, Kyoto, Japan) were used. The tumors were allowed to grow until they reached a diameter of about $0.5-1 \mathrm{~cm}$. The animals were allowed food and water ad libitum. The experiments were approved by the State of Bremen, Germany and were carried out in accordance with legal requirements.

\section{Biodistribution and histology}

Liposomes $(100 \mu \mathrm{L})$ were injected into the tail vein. After predetermined times, animals were sacrificed. For histology, organs were sampled and preserved immediately in $5 \%$ formalin in phosphate-buffered saline. For whole-body sectioning, ${ }^{16}$ the animals were frozen, embedded in 5\% carboxymethyl cellulose and sectioned with a whole-body cryomicrotome. The paraffin sections for histology were prepared in the usual way and stained with hematoxylin/eosin.

Neutron capture radiograms of whole-body sections of mice were prepared as described previously. ${ }^{16}$ The freezedried sections were placed against a Kodak Pathé LR115 film (Kodak Pathé, Paris, France) and irradiated with $4.10^{12} \mathrm{n} \mathrm{cm}^{-2}$ at Forschungsreaktor München II (Garching, Germany). The films were etched in $10 \% \mathrm{NaOH}$, until the holes penetrated the sensitive layer. 


\section{MRI}

The liposome solution (THF-S-16, 1:1:1 with cholesterol and DSPC, containing 2\% mol DSPE-PEG) was injected as a bolus of $100 \mu \mathrm{L}$ into the tail vein of a tumor-bearing $\mathrm{BALB} / \mathrm{c}$ mouse. Under anesthesia, the mouse was placed in prone position on an animal bed equipped with a nose cone for gas anesthesia ( $1.1 \%$ isoflurane in oxygen) and a temperature-controlled warming blanket in a 7T MRI system for small animals (BioSpec ${ }^{\circledR} 70 / 20$ USR; Bruker Corporation, Rheinstetten, Germany). In order to observe hemorrhage due to the destruction of blood vessels by the injected liposomes with MRI, T2*-weighted MRI data were acquired using a threedimensional gradient echo fast low-angle shot sequence ${ }^{17}$ with the following parameters: repetition time $=40$ milliseconds, echo time $=5$ milliseconds, excitation pulse width $=5$ degrees, field-of-view $=40 \times 20 \times 16 \mathrm{~mm}$, matrix $=192 \times 96 \times 32$, and image resolution $=208 \times 208 \times 500 \mu \mathrm{m}$. The first image for this time course was acquired 46 minutes after injection of the liposome solution, followed by a further 13 images over a time of 140 minutes. For image analysis, the MRI data were converted from the proprietary Bruker format to NIfTI format (NIfTI-1; National Institutes of Health, Bethesda, MD),${ }^{18}$ corrected for body motions using the "realign" function as implemented in SPM8 (Wellcome Trust Center for Neuroimaging, London, UK), ${ }^{19}$ and converted to a four-dimensional data set. Region-of-interest (ROI) based analysis was done on a selected single slice cutting through the hemorrhagic area using ImageJ software (National Institutes of Health, Bethesda, MD). ${ }^{20}$ Data represent the mean, with the error bars representing the standard error of the mean of signal intensities in the selected ROIs.

\section{Results}

The effects of four newly synthesized lipids, with three different basic structures, were tested in two different mouse tumor models. The structures of the lipids are shown in Figure 1. All the lipids contain a dodecaborate cluster as the head group, which carries two negative charges. The lipid B-6-14 has no group compensating part of the charge, whereas the other lipids have an additional positive charge at some distance from the hydrophobic part. These lipids have an oxapentane chain (THF lipids) or a bis(oxyethylene) chain (Dioxan lipids) between the cluster and the lipid moiety. All lipids can form bilayers (either open bilayers or closed vesicles) on their own. They can also form liposomes in combination with helper lipids. ${ }^{9} 10$ When prepared without DSPE-PEG, the
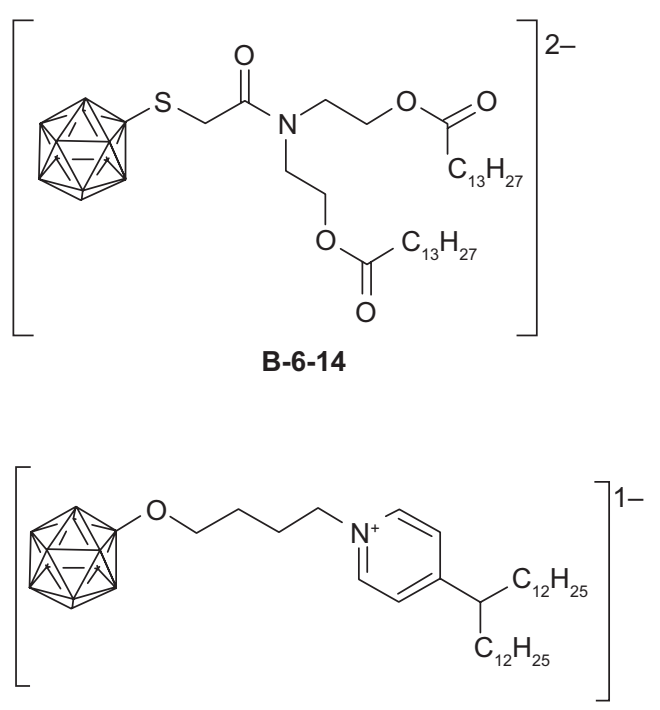

THF-S-12

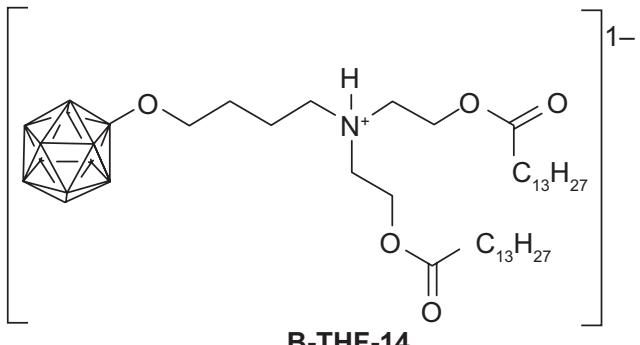

B-THF-14

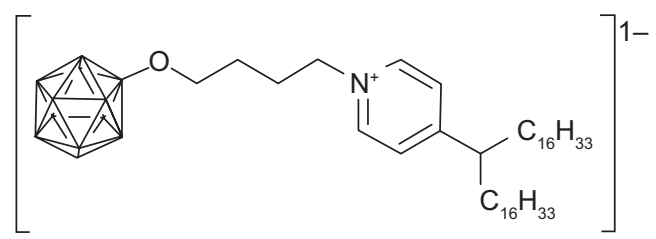

THF-S-16

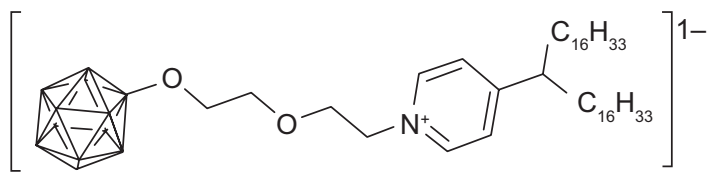

Dioxan-S-16

Figure I Structures of the lipids tested. In the icosahedra, each corner represents a boron atom, which carries an additional hydrogen atom if not substituted by an oxygen or sulfur atom. The dodecaborate cluster unit $\left(\mathrm{B}_{12} \mathrm{H}_{11}\right)$ carries a double negative charge, which is not shown for clarity. 
liposomes have $\zeta$-potentials of around $-45 \mathrm{mV}$ to $-60 \mathrm{mV}$, slightly dependent of the lipid used, while the addition of DSPE-PEG reduces this to $-5 \mathrm{mV}$, which is the value observed for liposomes prepared without a boron lipid. The sizes of the preparations were between $100-130 \mathrm{~nm}$, both in the absence and the presence of PEG lipids.

\section{Biodistribution in tumor models}

For the investigations in vivo, only lipids with median effective dose toxicity in vitro above $3 \mathrm{mM}$ were chosen. ${ }^{8-10}$

Two different tumor models were used for investigating the biodistribution: CT26.WT colon carcinoma (in BALB/c mice) and SCCVII murine squamous cell carcinoma (in $\mathrm{C} 3 \mathrm{H}$ mice). The liposomes consisted of the boron lipid, cholesterol, and DSPC in varying ratios, and all contained $5 \% \mathrm{~mol}$ DSPE-PEG. One lipid, B-THF-14, led to the death of two animals within 5 minutes after the injection and was therefore not investigated further. The other lipids, given in the same amounts of $20 \mathrm{mg}$ boron/ $\mathrm{kg}$ body weight, were well tolerated by the animals.

Upon whole-body cryosectioning of the animals injected with any of the lipids (THF-S-12, THF-S-16, Dioxan-S-16, or B-6-14), both of the tumor types were found to be dark red due to red blood cells; in contrast, the tumors in untreated animals consistently showed no macroscopically visible bleeding (Figure 2A-E). Hemorrhage occurred rapidly and was visible in the tumor in situ within about 1 hour.

In one animal to which THF-S-12 had been administered intravenously through the tail vein, death occurred within 2 hours after administration. Upon biopsy, it was found that some of the tumor cells had been injected unintentionally into the peritoneal cavity during subcutaneous implantation and had grown there, forming numerous tumor nodules. Upon biopsy, the peritoneum was found to be filled with blood; thus, the animal might have died from severe blood loss.

Neutron capture radiography ${ }^{16}$ showed that boron concentration was high in the liver and spleen (despite the fact that the liposomes were pegylated) (Figure 2F). The concentration of boron in the tumor was similar to that in blood, as expected from the large amount of erythrocytes found in the tumor. Uptake in the liver and spleen was considerably higher, in agreement with the experience of others with other boron lipids. ${ }^{11,21}$

\section{Histology}

Both types of tumors, injected with THF-S-12, THF-S-16, and Dioxan-S-16, were subjected to histological staining. In histology, pronounced hemorrhage was visible (Figure 3). The macroscopically visible red color of the tumors is most probably exclusively associated with extravasated red blood cells. Hemorrhage was classified as light, moderate, or heavy upon inspection of the microscopy slides (Figures 3A, 3B, and $3 \mathrm{C}$, respectively).

The induction of hemorrhage was found to be correlated to the total amount of injected boron lipid, and was only moderately affected by the presence of helper lipids (Table 2). In view of the invariably found hemorrhage at therapeutically relevant amounts given, no further systematic study was carried out, relating dose of boron lipids or their concentration in liposomes to the occurrence of hemorrhage.

There appeared to be a slight difference between the two tumor types as none of the prepared SCCVII exhibited

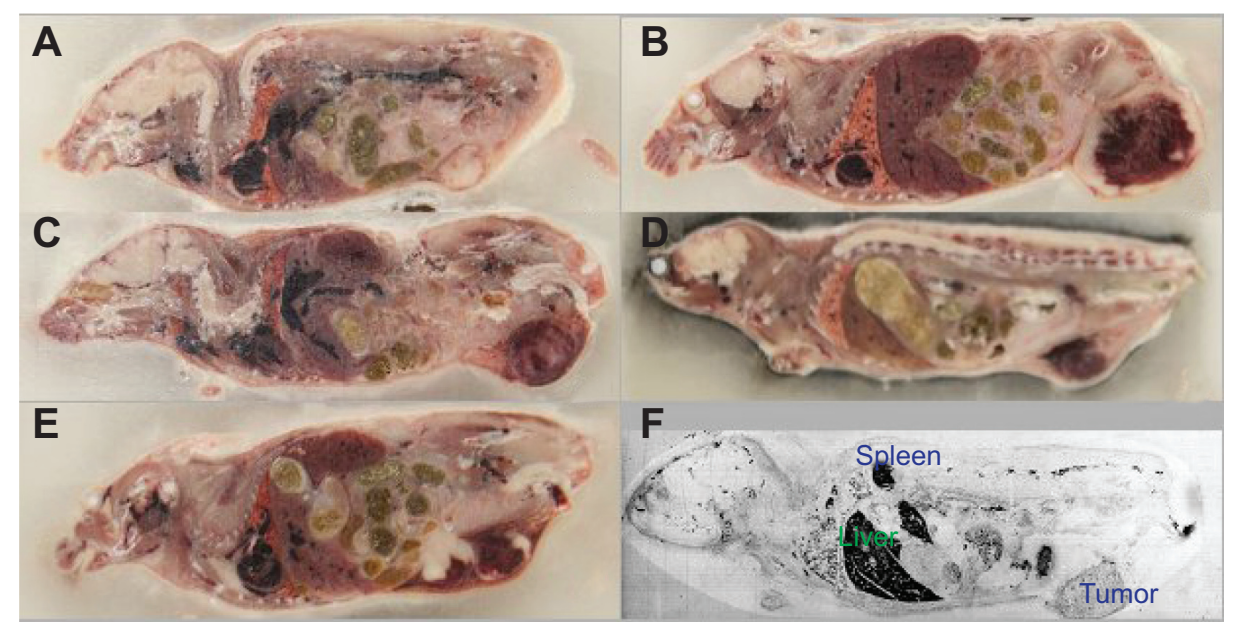

Figure 2 Photographs of mice embedded in carboxymethyl cellulose during cryosectioning.

Notes: (A) BALB/c without treatment; (B) BALB/c with THF-S- 123 hours after injection; (C) BALB/c with THF-S-16 22 hours after injection; (D) C3H with THF-S-I2 4 hours after injection; (E) BALB/c with B-6-14 20 hours after injection; and (F) neutron capture radiogram of BALB/c THF-S-12 4 hours after injection (tumor, spleen, and liver are labeled). High boron concentrations are indicated by a darker color. 


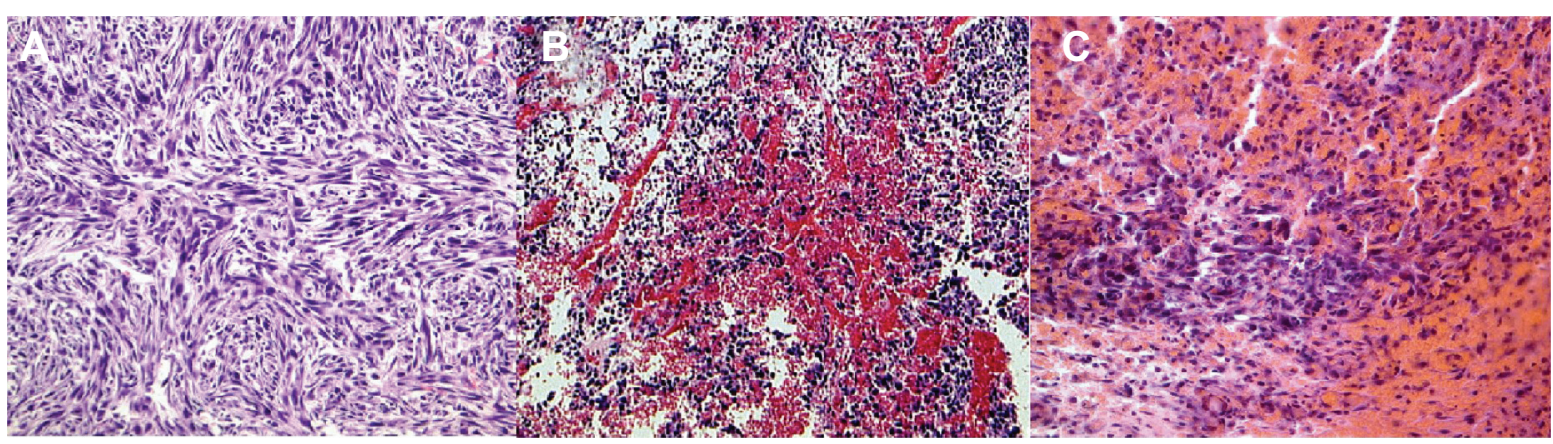

Figure 3 Photographs of histological sections of tumors. Sections were stained with hematoxylin/eosin.

Notes: (A) CT26.WT untreated; (B) SCCVII treated with THF-S-I2 (I30 $\mu \mathrm{g}$ boron per animal); and (C) CT26.WT treated with THF-S-I2 (I30 $\mu \mathrm{g}$ boron per animal), (B) was classified as moderate hemorrhage and $(\mathbf{C})$ as heavy hemorrhage.

heavy hemorrhage, only moderate hemorrhage at the highest dosages of boron lipid.

Other healthy tissues were examined and were not found to be affected by the liposomes.

\section{MRI}

Hemorrhage leads to a locally increased concentration of deoxygenated hemoglobin, decreasing the $\mathrm{T} 2 *$ relaxation in this area. Since gradient echo MRI methods are intrinsically sensitive for $\mathrm{T}^{*}$, a fast low-angle shot sequence ${ }^{17}$ was used.

In the area of the tumorous tissue, MRI revealed a signal decrease which was not observed in healthy muscle tissue serving as control. As depicted in Figure 4A, the signal decrease started around 100 minutes after injection of the liposomes (time point "b"). In contrast, the signal of healthy muscle tissue stayed constant, while in the tumorous tissue it decreased almost linearly during the next 90 minutes and leveled off afterwards. Figure 4B shows MRI at different time points with the corresponding ROIs of tumor and muscle tissue. The selected ROI of the tumor covered areas with and without hemorrhage; thus, the signal decrease would have appeared much more pronounced if only the hemorrhagic area would have been analyzed.
The onset of hemorrhage agrees with the visual observation of the tumor, where a strong reddening of the underlying tissue was observed after about 1 hour.

\section{Discussion}

The lipids used in this study all carry, as a hydrophilic headgroup, a dodecaborate cluster, with two negative charges. Different linkers between the negatively charged boron cluster and the hydrophobic moiety were used, and all lipids except B-6-14 carry an additional functional group with a positive charge under physiological conditions. B-THF-14 was toxic to the animals; its cytotoxicity was a little more pronounced than that of the other lipids (median effective dose of $0.38 \mathrm{mM}$ as compared to $1.5-4.8 \mathrm{mM}$ for the other lipids tested). The other boron lipids all led to the same effect - a massive hemorrhage in the tumors - despite the difference in their structures and the difference in the charge (B-6-14 carries two negative charges, whereas the others have only one negative net charge).

It is of special interest to note that all of the boron lipids had been tested for toxicity in cell culture before they were used in the animal models. The most cytotoxic boron lipid prepared by the authors was Dioxan-S-12 (similar to Dioxan-S-16 used here, but with only 12 carbon atoms in the

Table 2 Induction of hemorrhage by Dioxan-S-16 and THF-S-16

\begin{tabular}{llllll}
\hline B lipid & B:Cho:DSPC ratio & DSPC dose per animal $(\mu \mathrm{mol})$ & B $(\mu \mathrm{g})$ per animal & Degree of hemorrhage & Mouse \\
\hline Dioxan-S-16 & $0.5: 1: 1.5$ & 10 & 43 & Light & BALB/c \\
Dioxan-S-16 & $0.5: 1: 1.5$ & 10 & 43 & Light & C3H \\
Dioxan-S-16 & $1: 1: 1$ & 10 & 130 & Heavy & BALB/c \\
Dioxan-S-16 & $1: 1: 1$ & 10 & 130 & Moderate & C3H \\
Dioxan-S-16 & $0.25: 1: 1.75$ & 18 & 130 & Moderate & BALB/c \\
Dioxan-S-16 & $0.25: 1: 1.75$ & 18 & 130 & Moderate & C3H \\
THF-S-16 & $0.25: 1: 1.75$ & 5.8 & 22 & Little to light & BALB/c \\
THF-S-16 & $0.5: 1: 1.5$ & 5.0 & 43 & Light & BALB/c \\
THF-S-16 & $0.75: 1: 1.25$ & 4.2 & 65 & Moderate & BALB/c \\
\hline
\end{tabular}

Abbreviations: B, Boron; Cho, cholesterol; DSPC, distearoylphosphatidylcholine. 


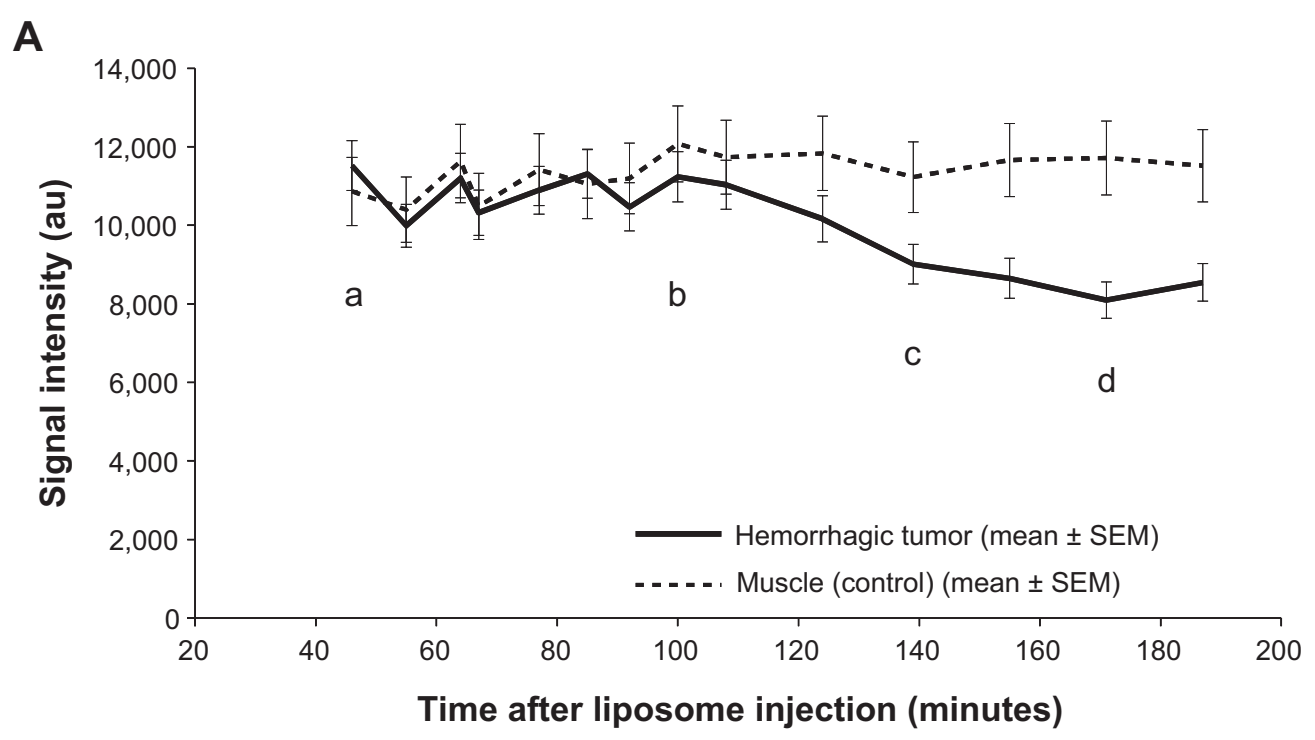

B
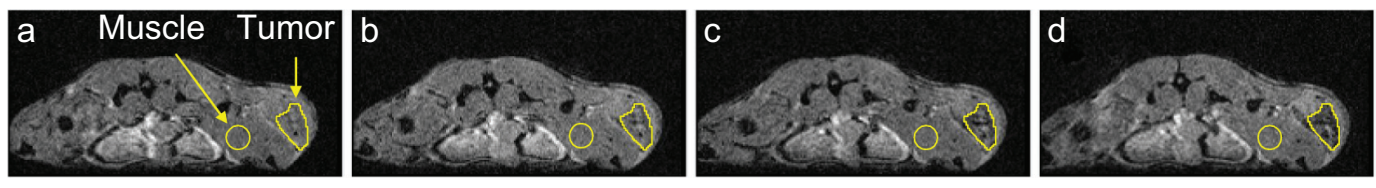

Figure 4 (A) Graphical representation of mean signal intensities \pm SEM of the tumor and normal muscle (control) tissue.

Notes: The development of hemorrhage is visualized by the signal loss (c-d). (B) Magnetic resonance images (fast low-angle shot) of regions of interest at selected time points (a-d) illustrating the signal changes plotted in the graph.

Abbreviation: SEM, standard error of the mean.

hydrophobic chain). ${ }^{10}$ While cytotoxicity is relatively easy to measure, it does not always appear to be a good predictor for in vivo effects. For example, B-THF-14 was toxic to animals within minutes, whereas the other lipids were tolerated well. Of the lipids used here, B-THF-14 was the most toxic in cell culture, with a toxicity similar to dioctadecyldimethylammonium bromide, commonly used for transfection experiments. ${ }^{22}$ For the other lipids, histology of selected healthy tissues (including the liver and spleen, where the highest boron concentrations are observed) showed no adverse effects. Therefore, the induction of hemorrhage in the tumors came as a surprise. For this study, a minimal number of animals were used, but the effect would not have been seen when relying on cell culture only, or when studying animals not carrying a tumor.

It was assumed that the massive hemorrhage observed was caused by destruction of the tumor blood vessels. Hemorrhage in the tumor could occur when only blood vessels in the tumor are destroyed selectively. Healthy tissue was not found to be affected by the boron lipids.

In the absence of DSPE-PEG, all of the lipids tested here resulted in liposomes which have a negative $\zeta$-potential (around $-45 \mathrm{mV}$ to $-60 \mathrm{mV}$ ). The PEG layer shields the charge to values of around $-25 \mathrm{mV}$. Nevertheless, if the assumption of selective damage to the tumor vasculature is correct, the liposomes appear to interact with the walls of the blood vessels, either with the basement membrane or the cells of the endothelial lining. Interaction of positively charged liposomes with tumor blood vessels is known. ${ }^{23}$

Selective destruction of blood vessels has been observed before by TNF $\alpha$ in combination with melphalan, ${ }^{24}$ by multiple doses of doxorubicin-containing liposomes, ${ }^{25}$ and by a combination of galactosamine and endotoxin. ${ }^{26}$ Overexpression of some isoforms of VEGF also leads to hemorrhage. ${ }^{26}$ As an induction of VEGF production would require at least several hours, this is not considered to be a likely cause of the effect observed with the boron-containing liposomes. One might hypothesize that the opening of the tumor blood vessel walls by the boron lipids could be caused by the induction of apoptosis in the cells of the endothelial lining of blood vessels in the tumor. ${ }^{27}$ This hypothesis is supported by the rather rapid onset of visible hemorrhage in the tumor, and the death of the one animal where the tumor had been inadvertently placed in the peritoneum. The hypothesis of induction of apoptosis will be investigated further, by looking for specific apoptosis markers in the tumors. Preliminary experiments have shown that liposomes containing these boron lipids are taken up by cells, and especially by human umbilical vein endothelial cells. 
Koganei et $\mathrm{a}^{21}$ have used a similar boron lipid in mice. Liposomes were prepared which contained both the boron lipids and BSH as a liposome-encapsulated BNCT agent, and neutron irradiation led to considerable reduction of the growth of tumors. The amount given to the animals (10 $\mu \mathrm{g}$ boron per gram body weight) was, however, not sufficient for a successful therapy in the absence of encapsulated BNCT agents, and only with the encapsulated water-soluble boron drug BSH, a therapeutic effect with BNCT could be observed. At the lowest dose tested in this work ( $22 \mu \mathrm{g}$ boron per gram body weight), very little hemorrhage was found. It can thus be assumed that in the experiments by Nakamura et al hemorrhage was not induced by the boron lipid due to the smaller amount administered in those experiments.

The pattern of uptake in healthy tissues of the boron lipids tested here is similar to that of other boron lipids described. ${ }^{14,21,28}$ Liver and spleen are the organs where most boron is found. For therapy, this is of no consequence if these organs are not exposed to the neutron beam.

Induction of hemorrhage in tumors has not been described with non-lipid boron compounds, even when the substances were toxic to animals. ${ }^{29-34}$ Therefore, it is assumed that the events leading to hemorrhage are caused by the specific amphiphilic character of the lipids used in this study.

The induction of tumor hemorrhage for BNCT is not a desired effect, even when the lipid would show very favorable distribution and retention patterns. For the lipids investigated here, tumor uptake was not very impressive, most probably caused by the rapid onset of hemorrhage and the filling of the tumor with blood, and thus these lipids would not be useful as boron carriers for BNCT.

The lipids investigated all carry an overall charge of -1 or -2 , and the charge might be responsible for the observed effect. The authors, therefore, intend to prepare boron-containing lipids with no net charge or a net charge of +1 to see whether such lipids also cause hemorrhage. If this would be the case, the observed effect might be caused by the properties of the boron cluster. They have recently been shown to exhibit an unusual pattern of hydration ${ }^{35}$ and were found to interact with carbohydrate chromatography matrices and lipid head groups attached to silica gel..$^{36,37}$ These findings might also have relevance for the development of non-lipid drugs for BNCT. If, however, hemorrhage would not be observed with neutral or positively charged dodecaborate lipids, such lipids might be used for accumulation of boron in tumors through the enhanced permeation and retention effect ${ }^{15}$ or with targeted liposomes. ${ }^{38}$

\section{Conclusion}

In conclusion, negatively charged lipids containing the dodecaborate anion as the headgroup have been investigated for in vivo pharmacodynamics in two animal models. All the boron lipids investigated for potential use in BNCT lead to hemorrhage in tumor tissue within the first 1-2 hours after application. As this phenomenon is undesired, their usage for BNCT deserves further discussion. Other strategies with different net charges using the same boron cluster, or with different clusters, must be tried for applying boron lipids successfully to BNCT.

\section{Acknowledgments}

The authors are grateful to Dr Ono, Kyoto University Research Reactor Institute, for giving them the SCCVII cells. They would like to thank Lipoid $\mathrm{GmbH}$ for the gift of distearoylphosphatidylcholine. This work was supported by the German Research Foundation DFG with a joint grant to D Gabel, R Süss, and R Schubert.

\section{Disclosure}

The authors report no conflicts of interest in this work.

\section{References}

1. Hawthorne MF. The role of chemistry in the development of boron neutron capture therapy of cancer. Angew Chem Int Ed Engl. 1993;32(7): 950-984.

2. Soloway AH, Tjarks W, Barnum BA, et al. The chemistry of neutron capture therapy. Chem Rev. 1998;98(4):1515-1562.

3. Fairchild RG, Bond VP. Current status of 10B-neutron capture therapy: enhancement of tumor dose via beam filtration and dose rate, and the effects of these parameters on minimum boron content: a theoretical evaluation. Int J Radiat Oncol Biol Phys. 1985;11(4):831-840.

4. Barth RF, Vicente MG, Harling OK, et al. Current status of boron neutron capture therapy of high grade gliomas and recurrent head and neck cancer. Radiat Oncol. 2012;7:146.

5. Nakamura H, Miyajima Y, Takei T, Kasaoka S, Maruyama K. Synthesis and vesicle formation of a nido-carborane cluster lipid for boron neutron capture therapy. Chem Commun (Camb). 2004;(17):1910-1911.

6. Nakamura H, Ueno M, Lee JD, et al. Synthesis of dodecaborateconjugated cholesterols for efficient boron delivery in neutron capture therapy. Tetrahedron Lett. 2007;48(18):3151-3154.

7. Lee JD, Ueno M, Miyajima Y, Nakamura H. Synthesis of boron cluster lipids: closo-dodecaborate as an alternative hydrophilic function of boronated liposomes for neutron capture therapy. Org Lett. 2007;9(2):323-326.

8. Justus E, Awad D, Hohnholt M, et al. Synthesis, liposomal preparation, and in vitro toxicity of two novel dodecaborate cluster lipids for boron neutron capture therapy. Bioconjug Chem. 2007;18(4):1287-1293.

9. Schaffran T, Lissel F, Samatanga B, et al. Dodecaborate cluster lipids with variable headgroups for boron neutron capture therapy: synthesis, physical-chemical properties, and toxicity. J Organomet Chem. 2009; 694(11):1708-1712.

10. Schaffran T, Burghardt A, Barnert S, et al. Pyridinium lipids with the dodecaborate cluster as polar headgroup: synthesis, characterization of the physical-chemical behavior, and toxicity in cell culture. Bioconjug Chem. 2009;20(11):2190-2198.

11. Li T, Hamdi J, Hawthorne MF. Unilamellar liposomes with enhanced boron content. Bioconjug Chem. 2006;17(1):15-20. 
12. Maruyama $\mathrm{K}$, Ishida $\mathrm{O}$, Kasaoka $\mathrm{S}$, et al. Intracellular targeting of sodium mercaptoundecahydrododecaborate $(\mathrm{BSH})$ to solid tumors by transferrin-PEG liposomes, for boron neutron-capture therapy (BNCT). J Control Release. 2004;98(2):195-207.

13. Nakamura H, Lee JD, Ueno M, Miyajima Y, Ban HS. Synthesis of closo-dodecaboryl lipids and their liposomal formation for boron neutron capture therapy. Nanobiotechnology. 2007;3(2):135-145.

14. Ueno M, Ban HS, Nakai K, et al. Dodecaborate lipid liposomes as new vehicles for boron delivery system of neutron capture therapy. Bioorg Med Chem. 2010;18(9):3059-3065.

15. Fang J, Nakamura $H$, Maeda $H$. The EPR effect: unique features of tumor blood vessels for drug delivery, factors involved, and limitations and augmentation of the effect. Adv Drug Deliv Rev. 2011;63(3):136-151.

16. Gabel D, Holstein H, Larsson B, et al. Quantitative neutron capture radiography for studying the biodistribution of tumor-seeking boroncontaining compounds. Cancer Res. 1987;47(20):5451-5454.

17. Frahm J, Haase A, Matthaei D. Rapid NMR imaging of dynamic processes using the FLASH technique. Magn Reson Med. 1986;3(2):321-327.

18. NIfTI-1. Bethesda, MD: National Institutes of Health; 2007. Available from: http://nifti.nimh.nih.gov/nifti-1/. Accessed June 11, 2014.

19. SPM8 software. London: Wellcome Trust Center for Neuroimaging; 2009. Available from: http://www.fil.ion.ucl.ac.uk/spm/software/spm8/. Accessed June 11, 2014.

20. ImageJ software. Bethesda, MD: National Institutes of Health; 1997. Available from: http://imagej.nih.gov/ij/. Accessed June 11, 2014.

21. Koganei H, Ueno M, Tachikawa S, et al. Development of high boron content liposomes and their promising antitumor effect for neutron capture therapy of cancers. Bioconjug Chem. 2013;24(1): 124-132.

22. Carmona-Ribeiro AM, Ortis F, Schumacher RI, Armelin MCS. Interactions between cationic vesicles and cultured mammalian cells. Langmuir. 1997;13(8):2215-2218.

23. Fasol U, Frost A, Buchert M, et al. Vascular and pharmacokinetic effects of EndoTAG-1 in patients with advanced cancer and liver metastasis. Ann Oncol. 2012;23(4):1030-1036.

24. Hoving S, Seynhaeve AL, van Tiel ST, et al. Early destruction of tumor vasculature in tumor necrosis factor- $\alpha$-based isolated limb perfusion is responsible for tumor response. Anticancer Drugs. 2006; 17(8):949-959.

25. Zhou R, Mazurchuk R, Straubinger RM. Antivasculature effects of doxorubicin-containing liposomes in an intracranial rat brain tumor model. Cancer Res. 2002;62(9):2561-2566.
26. Cheng SY, Nagane M, Huang HS, Cavenee WK. Intracerebral tumorassociated hemorrhage caused by overexpression of the vascular endothelial growth factor isoforms VEGF121 and VEGF165 but not VEGF189. Proc Natl Acad Sci U S A. 1997;94(22):12081-12087.

27. van Horssen R, Ten Hagen TL, Eggermont AM. TNF- $\alpha$ in cancer treatment: molecular insights, antitumor effects, and clinical utility. Oncologist. 2006;11(4):397-408.

28. Kueffer PJ, Maitz CA, Khan AA, et al. Boron neutron capture therapy demonstrated in mice bearing EMT6 tumors following selective delivery of boron by rationally designed liposomes. Proc Natl Acad Sci US A. 2013;110(16):6512-6517.

29. Feakes DA, Shelly K, Knobler CB, Hawthorne MF. Na3[B20H17NH3]synthesis and liposomal delivery to murine tumors. Proc Natl Acad Sci US A. 1994;91(8):3029-3033.

30. Slatkin DN, Micca PL, Forman A, Gabel D, Wielopolski L, Fairchild RG. Boron uptake in melanoma, cerebrum, and blood from Na2B12H11SH and Na4B24H22S2 administered to mice. Biochem Pharmacol. 1986;35(10):1771-1776.

31. Miura M, Micca PL, Heinrichs JC, Gabel D, Fairchild RG, Slatkin DN. Biodistribution and toxicity of 2,4-divinyl-nido-o-carboranyldeuteroporphyrin IX in mice. Biochem Pharmacol. 1992;43(3):467-476.

32. Haritz D, Gabel D, Huiskamp R. Clinical Phase-I study of $\mathrm{Na} 2 \mathrm{~B} 12 \mathrm{H} 1 \mathrm{SH}(\mathrm{BSH})$ in patients with malignant glioma as precondition for boron neutron capture therapy (BNCT). Int J Radiat Oncol Biol Phys. 1994;28(5):1175-1181.

33. Miura M, Joel DD, Smilowitz HM, et al. Biodistribution of copper carboranyltetraphenylporphyrins in rodents bearing an isogeneic or human neoplasm. J Neurooncol. 2001;52(2):111-117.

34. Miura M, Morris GM, Micca PL, et al. Boron neutron capture therapy of a murine mammary carcinoma using a lipophilic carboranyltetraphenylporphyrin. Radiat Res. 2001;155(4):603-610.

35. Karki K, Gabel D, Roccatano D. Structure and dynamics of dodecaborate clusters in water. Inorg Chem. 2012;51(9):4894-4896.

36. Fan P, Neumann J, Stolte S, et al. Interaction of dodecaborate cluster compounds on hydrophilic column materials in water. J Chromatogr A. 2012;1256:98-104.

37. Fan P, Stolte S, Gabel D. Interaction of organic compounds and boron clusters with new silica matrices containing the phosphatidylcholine headgroup. Anal Methods. 2014;6:3045-3055.

38. Ishida $\mathrm{O}$, Maruyama $\mathrm{K}$, Tanahashi $\mathrm{H}$, et al. Liposomes bearing polyethyleneglycol-coupled transferrin with intracellular targeting property to the solid tumors in vivo. Pharm Res. 2001;18(7):1042-1048.
International Journal of Nanomedicine

\section{Publish your work in this journal}

The International Journal of Nanomedicine is an international, peerreviewed journal focusing on the application of nanotechnology in diagnostics, therapeutics, and drug delivery systems throughout the biomedical field. This journal is indexed on PubMed Central, MedLine, CAS, SciSearch ${ }^{\circledR}$, Current Contents ${ }^{\circledR} /$ Clinical Medicine,
Dovepress

Journal Citation Reports/Science Edition, EMBase, Scopus and the Elsevier Bibliographic databases. The manuscript management system is completely online and includes a very quick and fair peer-review system, which is all easy to use. Visit http://www.dovepress.com/ testimonials.php to read real quotes from published authors. 